\title{
Optimized Kaiser-Bessel Window Functions for Computed Tomography
}

\author{
Masih Nilchian, Student Member, IEEE, John Paul Ward, Cédric Vonesch, and Michael Unser, Fellow, IEEE
}

\begin{abstract}
Kaiser-Bessel window functions are frequently used to discretize tomographic problems because they have two desirable properties: 1) their short support leads to a low computational cost and 2) their rotational symmetry makes their imaging transform independent of the direction. In this paper, we aim at optimizing the parameters of these basis functions. We present a formalism based on the theory of approximation and point out the importance of the partition-of-unity condition. While we prove that, for compact-support functions, this condition is incompatible with isotropy, we show that minimizing the deviation from the partition of unity condition is highly beneficial. The numerical results confirm that the proposed tuning of the Kaiser-Bessel window functions yields the best performance.
\end{abstract}

Index Terms - Kaiser-Bessel window function, approximation theory, tomography, inverse problem, generalized sampling.

\section{INTRODUCTION}

$\mathbf{I}$ T IS highly desirable to reduce the radiation dose in $\mathrm{X}$-ray imaging modalities. This can be achieved in two ways. The first solution involves a reduction in the intensity of the X-ray but contaminates the data with physical noise. The second solution involves a decrease in the number of projection angles. The price to pay for this reduction is that the reconstruction problem becomes ill-posed and can no longer be solved using traditional direct methods. Instead, the deployment of more sophisticated iterative schemes is needed. In order to specify such methods, one first discretizes the imaging operator and then selects a reconstruction scheme, which typically involves the choice of a cost functional to minimize. The second aspect is absolutely crucial when the problem is ill-posed and is typically addressed by introducing suitable regularization functionals such as sparsity or totalvariation regularization. This is the aspect of the problem that is addressed primarily in the literature [1]-[10].

Manuscript received October 13, 2014; revised March 6, 2015 and June 2, 2015; accepted June 5, 2015. Date of publication July 1, 2015; date of current version July 30, 2015. This work was supported in part by the European Research Council through the European Union's Seventh Framework Programme (FP7/2007-2013) under Grant 267439, in part by the Swiss National Science Foundation under Grant 200020-144355, and in part by the Center for Biomedical Imaging through the Geneva-Lausanne Universities and École Polytechnique Fédérale de Lausanne, Lausanne, Switzerland.

M. Nilchian, J. P. Ward, and M. Unser are with the Biomedical Imaging Group, École Polytechnique Fédérale de Lausanne, Lausanne 1015, Switzerland (e-mail: masih.nilchian@epfl.ch; jpward08@gmail.com; michael.unser@epfl.ch).

C. Vonesch is with the Center for Biomedical Imaging-SP, École Polytechnique Fédérale de Lausanne, Lausanne 1015, Switzerland (e-mail: cedric.vonesch@epfl.ch).

Color versions of one or more of the figures in this paper are available online at http://ieeexplore.ieee.org.

Digital Object Identifier 10.1109/TIP.2015.2451955
In this paper we want to concentrate on the first aspect: the choice of a suitable reconstruction space. This space is usually determined as a set of functions of the form

$$
f(\mathbf{x})=\sum_{\mathbf{k} \in \mathbb{Z}^{d}} c[\mathbf{k}] \varphi\left(\frac{\mathbf{x}}{T}-\mathbf{k}\right),
$$

where $T$ is the sampling step. The reconstruction space is then specified through the choice of the generating function $\varphi$.

In computed tomography, where the mathematical model is based on the Radon transform and its variants, it is beneficial to use a generating function that has two particular properties: 1) short support for fast computation and 2) rotational symmetry for efficient computation of the imaging transform. Among the functions satisfing these properties, Lewitt [11] introduced generalized Kaiser-Bessel window functions (KBWFs) as an optimal generating function to be used in the context of computed tomography. These functions are widely used in electron microscopy [12]-[14] and conventional X-ray and differential phase-contrast computed tomography [11], [15]-[18].

KBWFs involve three parameters that need to be adjusted [11]. These parameters are empirically tuned to improve the quality of reconstructed constant images [19], [20]. In this paper, we investigate approximationtheoretic properties of the basis functions, and we show how to optimize the parameters for the best performance. We also present experimental results that corroborate our theoretical prediction. The three contributions of this paper can be briefly summarized as follows:

- A measure to predict the performance of the generating function used for discretizing the forward model.

- A method to compute the optimal parameters for a generalized KBWF.

- An experimental validation of the proposed method.

The rest of this paper is organized as follows. In Section II, we describe the discretization scheme of the imaging operator and discuss the properties that should be satisfied by the generating function to be used for discretizing the forward model. In Section III, we study the KBWFs and propose new parameters for their use in the discretization scheme. Then, in Section IV, numerical experiments are presented to justify our choices.

\section{Discretization SCHEME}

We first explain how the discretization of the forward model is intimately connected with the choice of a given basis function. We then recall some fundamental results from 
approximation theory that ensure stability and allow one to predict the expected discretization error. This will point to the importance of the partition-of-unity property which, unfortunately as we shall prove, is incompatible with the compact-support and isotropy properties.

\section{A. Matrix Formulation}

Reconstruction is usually formulated as a linear inverse problem. To solve it, it is convenient to introduce discrete representations of the object and the imaging operator. Without loss of generality, we consider an object in 2D. The model of the object, from the perspective of generalized sampling theory [21], [22], is obtained by specifying a suitable reconstruction space. Specifically, we select $V_{T}(\varphi)$ as the principal shift-invariant space generated by the function $\varphi \in L_{2}\left(\mathbb{R}^{2}\right)$. This space is defined by

$$
V_{T}(\varphi)=\left\{\sum_{\mathbf{k} \in \mathbb{Z}^{2}} c[\mathbf{k}] \varphi\left(\frac{\mathbf{x}}{T}-\mathbf{k}\right): c \in \ell_{2}\left(\mathbb{Z}^{2}\right)\right\},
$$

where $\mathbf{x} \in \mathbb{R}^{2}$. The corresponding orthogonal projection operator $\mathbf{P}_{T}: L_{2}\left(\mathbb{R}^{2}\right) \rightarrow V_{T}(\varphi)$ is defined as

$$
\mathbf{P}_{T} f=\underset{g \in V_{T}(\varphi)}{\operatorname{argmin}}\|f-g\|_{L_{2}} .
$$

In practice, however, the values of $c$ in (1) are determined based on the solution of an inverse problem.

The mathematical model of many imaging modalities in the context of X-ray computed tomography is based on the Radon transform and its derivatives. Note that the Radon transform is a linear and pseudo shift-invariant operator,

$$
\mathcal{R}\{\varphi(\mathbf{x}-\mathbf{k})\}(y, \theta)=\mathcal{R}\{\varphi(\mathbf{x})\}(y-\langle\mathbf{k}, \boldsymbol{\theta}\rangle, \theta),
$$

where $\boldsymbol{\theta}=(\cos \theta, \sin \theta)$. Then, its application on a function $f \in V_{T}(\varphi)$ is

$$
\begin{aligned}
\mathcal{R}^{(n)}\{f\}(y, \theta) & =\sum_{\mathbf{k} \in \mathbb{Z}^{2}} c[\mathbf{k}] \mathcal{R}^{(n)}\left\{\varphi_{T}(\mathbf{x}-T \mathbf{k})\right\}(y, \theta) \\
& =\sum_{\mathbf{k} \in \mathbb{Z}^{2}} c[\mathbf{k}] \mathcal{R}^{(n)}\left\{\varphi_{T}\right\}(y-T\langle\mathbf{k}, \boldsymbol{\theta}\rangle, \theta),
\end{aligned}
$$

where $\varphi_{T}(\mathbf{x})=\varphi(\mathbf{x} / T)$, and

$$
\mathcal{R}^{(n)} f(y, \theta)=\frac{\partial^{n} \mathcal{R} f}{\partial y^{n}}(y, \theta),
$$

with $\mathcal{R}: L_{2}\left(\mathbb{R}^{2}\right) \rightarrow L_{2}(\mathbb{R} \times[0, \pi])$ being the Radon-transform operator.

The formulation of the reconstruction as a linear inverse problem is usually stated as the matrix equation

$$
\mathbf{g}=\mathbf{H c},
$$

where $\mathbf{g}$ is the measurement vector, $\mathbf{H}$ is the system matrix, and $\mathbf{c}$ is the discrete representation of the object of interest.

Using (5), the matrix formulation can be obtained as follows: The measurement vector $\mathbf{g}$ contains values of the imaging transform $\mathcal{R}^{(n)}\{f\}(y, \theta)$ at the sampled points $y_{j}=j \Delta y$ and $\theta_{i}=i \Delta \theta$, where $i, j \in \mathbb{Z}$. The object $f$ is represented with its coefficients $c$ within the space $V_{T}(\varphi)$. The system matrix $\mathbf{H}$ is given by

$$
[\mathbf{H}]_{(i, j), \mathbf{k}}=\mathcal{R}^{(n)}\left\{\varphi_{T}\right\}\left(y_{j}-T\left\langle\mathbf{k}, \boldsymbol{\theta}_{i}\right\rangle, \theta_{i}\right) .
$$

Note that, in order to compute the imaging operator, there is no need to store the whole system matrix because it is sufficient to have access to a lookup table that contains the projection of one basis function along every direction.

\section{B. Desirable Properties of the Basis Functions}

We require the basis function $\varphi$ to satisfy the following four properties:

1) Riesz Basis: Every object $f \in V_{T}(\varphi)$ must be uniquely specified by its coefficients $c$. This requires the existence of a positive constant $A$ such that

$$
\forall c \in \ell_{2}, \quad A \cdot\|c\|_{\ell_{2}}^{2} \leq\left\|\sum_{\mathbf{k} \in \mathbb{Z}^{2}} c[\mathbf{k}] \varphi\left(\frac{\mathbf{x}}{T}-\mathbf{k}\right)\right\|_{L_{2}} .
$$

In addition, the representation should be stable. This requires the existence of a positive constant $B$ such that

$$
\forall c \in \ell_{2}, \quad\left\|\sum_{\mathbf{k} \in \mathbb{Z}^{2}} c[\mathbf{k}] \varphi\left(\frac{\mathbf{x}}{T}-\mathbf{k}\right)\right\|_{L_{2}} \leq B \cdot\|c\|_{\ell_{2}}^{2} .
$$

Together, these two conditions are equivalent to $\varphi$ being a Riesz basis of $V_{T}(\varphi)$.

2) Partition of Unity: It is constructive for such a discretization scheme that the model approximate any input function as closely as desired by choosing a sufficiently small sampling step. More precisely, the approximation error should vanish whenever the sampling step $T$ tends to zero. We thus require that

$$
\lim _{T \rightarrow 0}\left\{\left\|f-\mathbf{P}_{T} f\right\|_{L_{2}}\right\}=0 .
$$

Theorem 1 [23]: The $L_{2}$-approximation error of the operator $\mathbf{P}_{T}: L_{2} \rightarrow V_{T}(\varphi)$ can be written, for $f \in L_{2}\left(\mathbb{R}^{2}\right)$, by

$$
\begin{aligned}
\epsilon_{f}(T) & =\left\|f-\mathbf{P}_{T} f\right\|_{L_{2}} \\
& =\left(\int_{\mathbb{R}^{2}} E_{\varphi}(T \omega)|\widehat{f}(\omega)|^{2} \frac{\mathrm{d} \omega}{2 \pi}\right)^{1 / 2}+\epsilon_{\mathrm{corr}},
\end{aligned}
$$

where $\epsilon_{\text {corr }}$ is a correction term, and $E_{\varphi}$ is the error kernel defined in the least-squares case as

$$
E_{\varphi}(\omega)=1-\frac{|\widehat{\varphi}(\omega)|^{2}}{\sum_{\mathbf{k} \in \mathbb{Z}^{2}}|\widehat{\varphi}(\omega+2 \mathbf{k} \pi)|^{2}},
$$

where $\widehat{\varphi}$ is the Fourier transform of $\varphi$. Specifically, if $f \in W_{2}^{r}$ (Sobolev space of order $r$ ) with $r>1 / 2$, then $\left|\epsilon_{\text {corr }}\right|<\gamma T^{r}\left\|f^{(r)}\right\|_{L_{2}}$, where $\gamma$ is some constant.

Under the regularity assumption that $f$ and its first order derivative are in $L_{2}\left(\mathbb{R}^{2}\right)$, the asymptotic convergence

$$
\lim _{T \rightarrow 0} \epsilon_{f}(T)=0
$$

is achieved if and only if the basis function $\varphi$ satisfies the partition-of-unity condition [21, Appendix B]

$$
\sum_{\mathbf{k} \in \mathbb{Z}^{2}} \varphi(\mathbf{x}+\mathbf{k})=1, \quad \forall \mathbf{x} \in \mathbb{R}^{2} .
$$


The equivalent formulation of the partition of unity in the frequency domain is

$$
\widehat{\varphi}(2 \pi \mathbf{n})=\delta[\mathbf{n}], \quad \forall \mathbf{n} \in \mathbb{Z}^{2},
$$

where $\delta$ is the 2D Kronecker delta function.

3) Compact Support: The basis function $\varphi$ should be compactly supported in order to reduce the computational cost and also for localization in the spatial domain.

4) Isotropy: For the implementation of the imaging operator, it is required to store the values of its application on the basis function along different directions. If the basis function is isotropic, its projections do not depend on the direction, which leads to simplicity and efficiency of implementation.

\section{Incompatible Properties}

There is a negative result that is considered in the following theorem:

Theorem 2: The following properties are mutually exclusive for an isotropic basis function:

1) compact support;

2) partition of unity.

Proof: The proof is given in Appendix A. Here, we provide only a sketch of the argument. The partition-of-unity condition implies the configuration (15) of zeros of the Fourier transform of the basis function. At the same time, the Hankel transform of an even compactly supported function is an entire function of finite exponential type. Jensen's theorem provides a contradiction between these two properties.

\section{Revisiting Optimality in the Projection Domain}

We now bound the error of approximation incurred by $\mathcal{R}_{T} f=\mathcal{R}\left\{\mathbf{P}_{T} f\right\}$. It can be extended to any derivative of the Radon transform through the Fourier-slice theorem since $\left\|\mathcal{R}^{(n)} f\right\|_{L_{2}}=\left\|\mathcal{R} \mathcal{F}^{-1}\left\{|\omega|^{n} \widehat{f}(\boldsymbol{\omega})\right\}\right\|_{L_{2}}$. To this end, we use the Sobolev norm $\|\cdot\|_{W_{2}^{1 / 2}}^{2}$ in the projection domain. If $g \in L_{2}\left(\mathbb{R}^{2}\right)$, then

$$
\|g\|_{W_{2}^{1 / 2}}^{2}=\int_{0}^{2 \pi} \int_{0}^{\infty}\left(1+\omega^{2}\right)^{\frac{1}{2}}|\widehat{g}(\omega, \theta)| \mathrm{d} \theta \mathrm{d} \omega,
$$

where $\widehat{g}(\omega, \theta)$ is the polar form of the Fourier transform of $g$.

Theorem 3: Let the Sobolev approximation error of the operator $\mathcal{R}_{T}$ for $f \in L_{2}\left(\mathbb{R}^{2}\right)$ be $\epsilon_{\mathcal{R} f}(T)=$

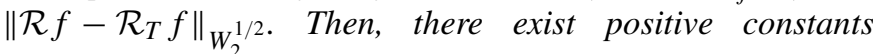
$r_{1}, R_{1}>0$ such that

$$
r_{1} \epsilon_{f}(T) \leq \epsilon_{\mathcal{R} f}(T) \leq R_{1} \epsilon_{f}(T) .
$$

Lemma [25, Sec. II.5]: Let $\Omega \subset \mathbb{R}^{2}$ be a compact domain. Then, there exist positive constants $r_{2}$ and $R_{2}$ such that, for any $L_{2}\left(\mathbb{R}^{2}\right)$ function $f$ that is supported on $\Omega$, it holds that

$$
r_{2}\|f\|_{L_{2}} \leq\|\mathcal{R} f\|_{W_{2}^{1 / 2}} \leq R_{2}\|f\|_{L_{2}} .
$$

Proof of Theorem 3: By letting $f \leftarrow\left(f-\mathbf{P}_{T} f\right)$ in (18), we obtain (17).

This theorem implies that the average error over all angles is small in the transform domain when the error of approximation is small in the object domain. While the theorem is an average result that involves a continuum of angles, it is still useful practically because it gives us the approximation error in the transform domain over a family of images that would correspond to all rotated versions of a given reference image.

\section{Optimized KAISER-BesSEL Window Function}

The generalized family of KBWFs is isotropic, which makes it advantageous for the representation of the imaging operator. Our goal here will be to determine the optimal set of parameters to best match the partition-of-unity condition which is so fundamental to approximation theory.

\section{A. Generalized Kaiser-Bessel Window Functions}

The generalized KBWF, defined as

$$
\varphi(\mathbf{x})= \begin{cases}\frac{\left(\sqrt{1-(\|\mathbf{x}\| / a)^{2}}\right)^{m} I_{m}\left(\alpha \sqrt{1-(\|\mathbf{x}\| / a)^{2}}\right)}{I_{m}(\alpha)} & 0 \leq\|\mathbf{x}\| \leq a \\ 0 & \text { otherwise }\end{cases}
$$

is specified by three parameters: 1 ) the order $m$ of the modified Bessel function $I_{m} ; 2$ ) the window taper $\alpha$; 3) the support radius $a$ of the function. The parameter $m$ allows us to control the smoothness of the function and the parameter $\alpha$ determines its shape. This function is isotropic, which makes the computation of the imaging operator significantly faster. However, it is worth noting that this function does not satisfy the partition of unity (see Theorem 2).

\section{B. Measure of Optimality of a Basis Function}

If a basis function satisfies the partition-of-unity condition, then, as the sampling step vanishes, the error of approximation tends to zero. For those bases that do not satisfy the partition of unity, we define the residual error

$$
A_{\varphi}=\sup _{f \in L_{2}}\|f\|_{L_{2}}^{-1} \lim _{T \rightarrow 0} \epsilon_{f}(T)
$$

for $f \in L_{2}\left(\mathbb{R}^{d}\right)$, which shows the deviation from the partition of unity. A basis function $\varphi$ with lower residual error is more desirable as a generating function for the reconstruction space.

Theorem 4: The residual error of a function $\varphi \in L_{2}\left(\mathbb{R}^{d}\right)$ is the quantity

$$
A_{\varphi}=\frac{\sum_{\mathbf{n} \neq \mathbf{0}}|\widehat{\varphi}(2 \pi \mathbf{n})|^{2}}{|\widehat{\varphi}(\mathbf{0})|^{2}} .
$$

Proof: We assume the regularity condition that $f$ and its first order derivative are in $L_{2}$. From (11), we have a formula for $\epsilon_{f}$ in terms of $E_{\varphi}$ as defined in (12). We represent $E_{\varphi}$ using its Taylor series

$$
E_{\varphi}(T \boldsymbol{\omega})=\sum_{|\mathbf{n}|=0}^{N} \frac{\partial^{\mathbf{n}} E_{\varphi}(\mathbf{0})}{\mathbf{n} !}(T \boldsymbol{\omega})^{\mathbf{n}}+o\left(\left\|T^{N+1} \boldsymbol{\omega}\right\|^{N+1}\right),
$$

where $\mathbf{n}=\left(n_{1}, n_{2}, \ldots, n_{d}\right)$ with nonnegative integer values, $|\mathbf{n}|=\sum_{i=1}^{d} n_{i}, \boldsymbol{\omega}=\left(\omega_{1}, \omega_{2}, \ldots, \omega_{d}\right), \mathbf{n} !=n_{1} ! n_{2} ! \ldots n_{d} !$, $\boldsymbol{\omega}^{\mathbf{n}}=\omega_{1}^{n_{1}} \omega_{2}^{n_{2}} \ldots \omega_{d}^{n_{d}}$, and

$$
\partial^{\mathbf{n}} E_{\varphi}(\mathbf{0})=\frac{\partial^{n_{1}}}{\omega_{1}} \frac{\partial^{n_{2}}}{\omega_{2}} \cdots \frac{\partial^{n_{d}}}{\omega_{d}} E_{\varphi}(\mathbf{0}) .
$$


Therefore, we can rewrite the approximation error $\epsilon_{f}$ as

$$
\begin{aligned}
\epsilon_{f}(T) & =\left\|f-\mathbf{P}_{T}\{f\}\right\|_{L_{2}} \\
& =\left(\int_{\mathbb{R}^{d}}\left(\sum_{|\mathbf{n}|=0}^{N} \frac{\partial^{\mathbf{n}} E_{\varphi}(\mathbf{0})}{\mathbf{n} !}(T \boldsymbol{\omega})^{\mathbf{n}}\right)|\widehat{f}(\boldsymbol{\omega})|^{2} \frac{\mathrm{d} \boldsymbol{\omega}}{2 \pi}\right)^{1 / 2}+\epsilon,
\end{aligned}
$$

where $\epsilon=o\left(T^{N+1}\|\omega\|^{N+1}\right)+\epsilon_{\text {corr }}$. Then, Fubini's theorem implies that

$$
\begin{aligned}
\epsilon_{f}(T) & =\left(\sum_{|\mathbf{n}|=0}^{N} \frac{\partial^{\mathbf{n}} E_{\varphi}(\mathbf{0})}{\mathbf{n} !} T^{|\mathbf{n}|} \int_{\mathbb{R}^{d}} \omega^{\mathbf{n}}|\widehat{f}(\boldsymbol{\omega})|^{2} \frac{\mathrm{d} \boldsymbol{\omega}}{2 \pi}\right)^{1 / 2}+\epsilon \\
& =\left(\sum_{|\mathbf{n}|=0}^{N} \frac{\partial^{\mathbf{n}} E_{\varphi}(\mathbf{0})}{\mathbf{n} !} T^{|\mathbf{n}|}\left\|f^{(\mathbf{n} / 2)}\right\|_{L_{2}}^{2}\right)^{1 / 2}+\epsilon,
\end{aligned}
$$

where

$$
f^{(\mathbf{n})}=\frac{\partial^{n_{1}}}{\partial x_{1}} \frac{\partial^{n_{2}}}{\partial x_{2}} \cdots \frac{\partial^{n_{d}}}{\partial x_{d}} f .
$$

We now have that

$$
\lim _{T \rightarrow 0} \epsilon_{f}(T)=E_{\varphi}(\mathbf{0})^{1 / 2}\|f\|_{L_{2}} .
$$

Therefore,

$$
\begin{aligned}
\sup _{f \in L_{2}}\|f\|_{L_{2}}^{-2}\left(\lim _{T \rightarrow 0} \epsilon_{f}(T)\right)^{2} & =\sup _{f \in L_{2}} E_{\varphi}(\mathbf{0}) \\
& =\frac{\sum_{\mathbf{n} \neq \mathbf{0}}|\widehat{\varphi}(2 \pi \mathbf{n})|^{2}}{|\widehat{\varphi}(\mathbf{0})|^{2}} .
\end{aligned}
$$

\section{Optimal Parameters for the Kaiser-Bessel Window Function}

There are three parameters that describe KBWFs. The radius parameter $a$ determines its support. We set it to $a=2$; this allows us to compare the optimal KBWF with the cubic B-spline. The order of the modified Bessel function is set to $m=2$.

In the context of 3D imaging, Matej and Lewitt [19] empirically tune the window taper parameter $\alpha$ to improve the quality of reconstructed constant images. In contrast, we base our analysis on approximation-theoretic properties and determine $\alpha$ to minimize the residual error $A_{\varphi}$. Interestingly, this leads to a condition similar to the complicated criterion of [19]. But we go one step farther and provide a simplified equivalent condition in (21). The measure for different values $\alpha$ is depicted in Fig. 1(b). This plot indicates that values of $\alpha$ in the range $[6,11.2]$ are good choices for reconstruction, with two local optima of $\alpha=7.05,10.45$ of comparable magnitude. The latter value is very close to 10.4 , which is the value proposed in [19].

There are modalities where the reconstruction problem is separable into a set of independent 2D problems: X-ray parallel-beam tomography, transmission electron microscopy with single-axis tilting, 2D positron emission tomography systems with septa, and single-photon emission computed
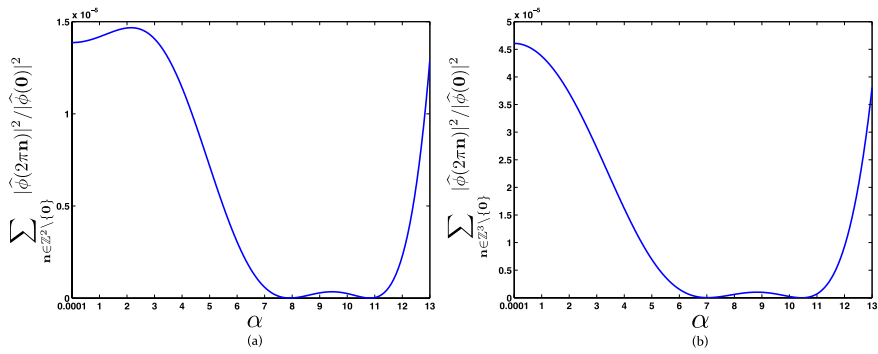

Fig. 1. The Kaiser-Bessel window taper parameter is denoted by $\alpha$ in the Figure. Optimality measure with respect to different values of $\alpha$ in the (a) $2 \mathrm{D}$ and (b) $3 \mathrm{D}$ domains.

tomography with parallel or fan-beam collimators. Then it is worthwhile to evaluate the optimal parameters for 2D KBWFs. We illustrate in Fig. 1(a) the residual error with respect to the parameter $\alpha$ in a 2D space. Again, it appears that values of $\alpha$ in the range $[7,11.5]$ are good choices for $2 \mathrm{D}$ reconstruction, with $\alpha=7.91,10.83$ being the two best choices.

\section{Numerical EVAluation}

We now present experiments where we numerically evaluate the discretization scheme based on KBWFs, with the parameters suggested in this paper.

\section{A. Influence of the Discretization Step}

By definition the optimal reconstruction in the least-square sense is the orthogonal projection of the sample on the reconstruction space, independently of the chosen algorithm. To investigate the dependence upon the grid size, we compute the optimal reconstruction with respect to different grid sizes when the generating function of the reconstruction space is a KWBF with different parameters. The reference object and signal-to-noise (SNR) computations are defined with respect to the fine grid. The SNR is defined as the relative mean-square with respect to the reference (oracle),

SNR(oracle,reconstruction)

$$
=20 \log \left(\frac{\| \text { oracle } \|_{L_{2}}}{\| \text { oracle-reconstruction } \|_{L_{2}}}\right) .
$$

The grid size is progressively increased, which shows the dependences upon the sampling rate.

We choose two medical samples: a coronal section of a human lung and a coronal section of a rat brain. Also, a region of interest has been chosen as shown in Figs. 2(a) and 2(b). We first tested the KBWF with $\alpha=2$, which is well outside of the optimal interval $[7,11.5]$, and the results were very poor $(\mathrm{SNR}=4 \mathrm{~dB})$. We then compared the performance for the value $\alpha=5$ and $\alpha=7.91$. The former is close to, but outside of the optimal interval, while the latter is the first of our proposed choices. Their performances are depicted in Figs. 2(c) and 2(d). It confirms that using KBWFs with the proposed parameter has better optimal reconstruction compared to $\alpha=5$ for different grid sizes. This experiment shows that the "optimal choice" based on the asymptotical behavior (see (20)) is also always better for different grid sizes. The results for four times coarser grid representation 


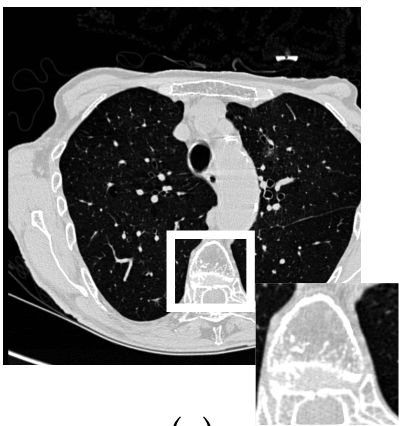

(a)

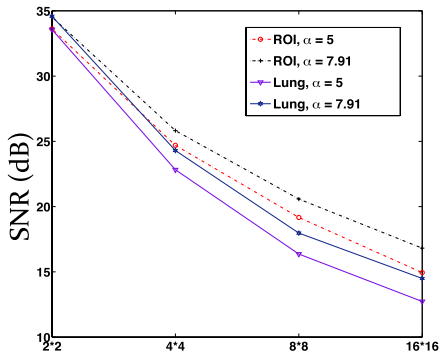

(c)

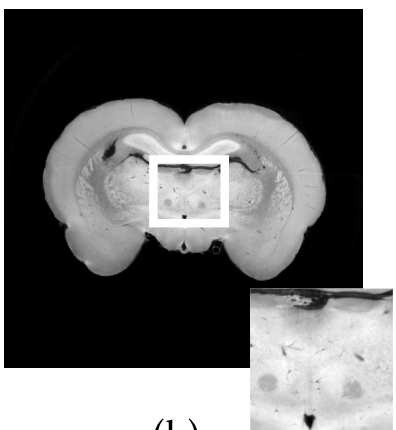

(b)

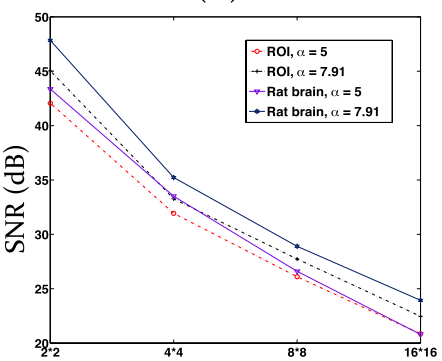

(d)
Fig. 2. (a) Coronal section of a human lung and region of interest (b) Coronal section of a rat brain and region of interest. The performance of the optimal solution with respect to the grid size is depicted in (c) and (d).

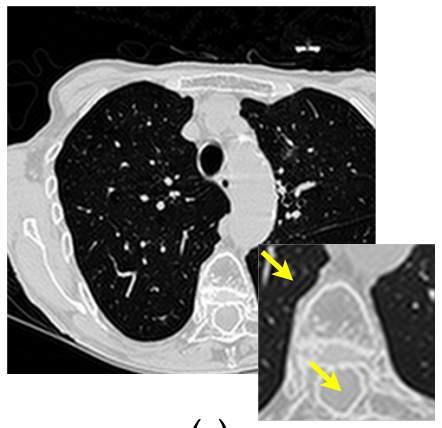

(a)

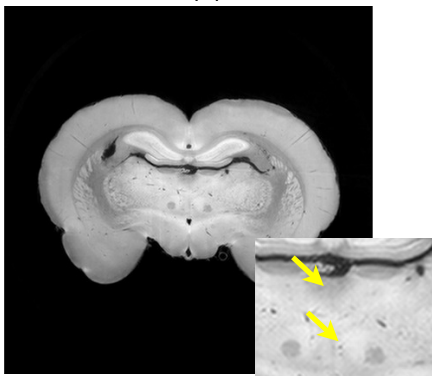

(c)

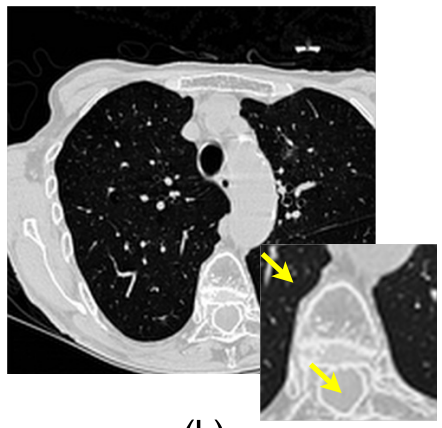

(b)

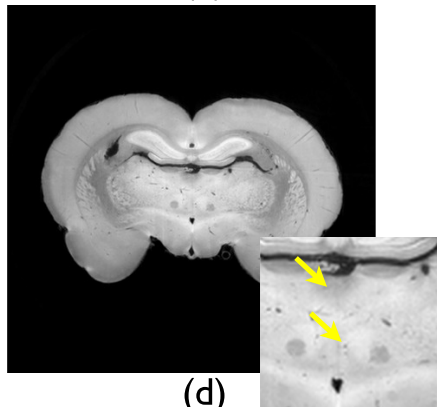

(d)

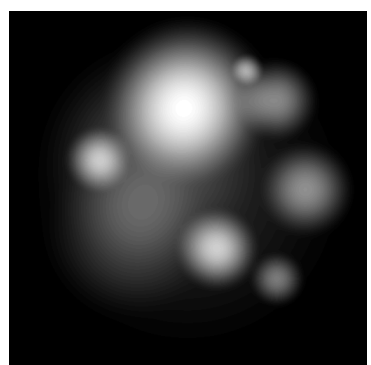

(a)

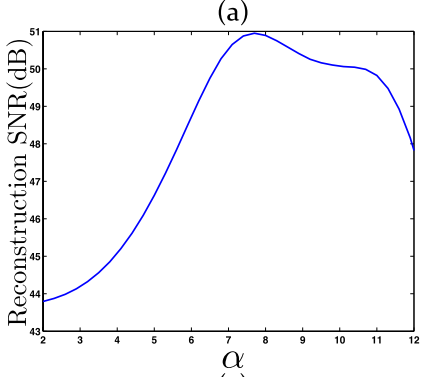

(c)

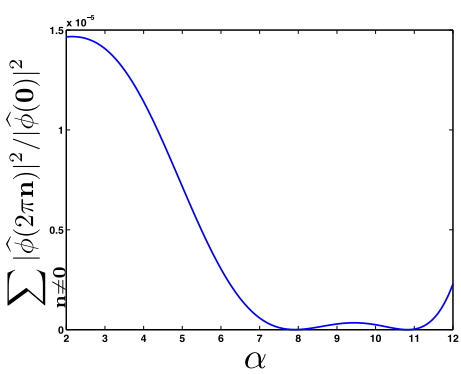

(b)

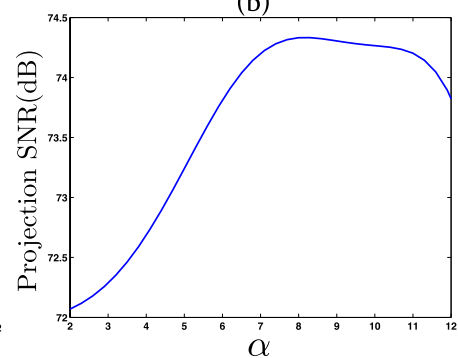

(d)
Fig. 4. The Kaiser-Bessel window taper parameter is denoted by $\alpha$ in the Figure. (a) 2D analytical phantom with isotropic elements. (b) Zoomed version of the proposed measure. The accuracy of the reconstruction of the analytical phantom versus the window taper parameter of KBWFs is shown in (c). Its Radon- transform error in the same coarse grid is depicted in (d).

1) Conventional Tomography: The size of the phantom for the first experiment is $(2,048 \times 2,048)$ pixels. The sinogram of the phantom is computed analytically with 1,800 viewing angles that are chosen uniformly between 0 and $\pi$; it comprises the measurements. Note that the resolution of the reconstruction in the fine grid is the same as the resolution of the measurements (detector pixel size). As there is a large number of views for the reconstruction, we minimize the leastsquares error

$$
J(\mathbf{c})=\|\mathbf{H c}-\mathbf{g}\|_{L_{2}}^{2},
$$

where $\mathbf{g}$ is the measurement vector.

The object is reconstructed on a grid that is $(4 \times 4)$ times coarser than the discretization grid. Then, the basis function is used to resample the object on a finer grid. We use the conjugate-gradient algorithm for the minimization. As the number of directions is on the order of the size of the object, we do not use any regularization. The signal-to-noise ratio (SNR) of the reconstructions and the projection versus different values of the window taper of KBWFs are shown in Fig. 4(c) and 4(d). The best performance is obtained by using a KBWF with $\alpha=7.75$, which is very close to the first minimum of our criterion function in Fig. 1. However, values of $\alpha$ in the range $[7,11.5]$ do also perform reasonably well, which is consistent with theoretical analysis of Section III-C.

2) Differential Phase-Contrast Tomography: We evaluate the performance of KBWFs with the proposed parameters in $\mathrm{X}$-ray differential phase-contrast tomography. The mathematical model of this imaging modality is based on the derivative of the Radon transform.

The differentiated sinogram of the phantom with size $(512 \times 512)$ pixels is again computed analytically

with 1,800 viewing angles that are chosen uniformly and shown in Fig. 4. (a). The analytical formula for computing imaging transforms of the phantom is given in [7, Sec. 4.4]. 


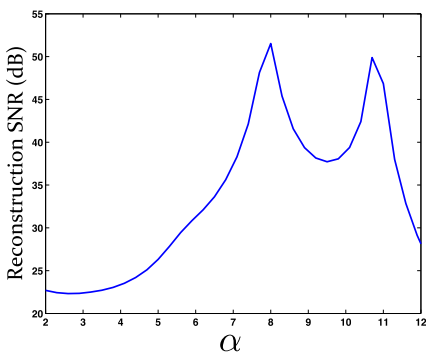

(a)

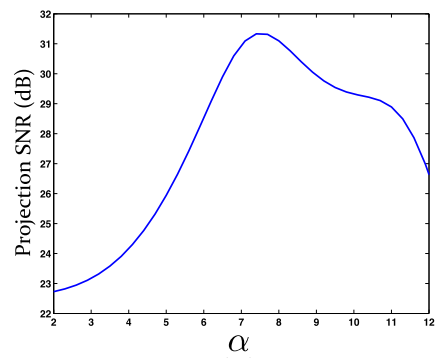

(b)
Fig. 5. The Kaiser-Bessel window taper parameter is denoted by $\alpha$ in the Figure. Performance of the (a) reconstruction and (b) projection using KBWFs for differential phase-contrast tomography versus the window taper parameter.

TABLE I

COMPARISON OF THE PROJECTION AND RECONSTRUCTION ACCURACY Using CUbic B-Splines AND KBWFs With the Parameters PROPOSED IN [18]

\begin{tabular}{l|ccc|c}
\hline \hline SNR (dB) & \multicolumn{3}{|c|}{ KBWF $a=2}$, & Cubic \\
& \multicolumn{3}{|c}{$m=2}$, & B-spline \\
& $\alpha=2$ & $\alpha=10.4$ & $\alpha=7.95$ & \\
\hline Projection & 23.59 & 27.15 & 29.19 & 29.26 \\
Reconstruction & 37.31 & 43.42 & 48.59 & 48.14 \\
\hline
\end{tabular}

between 0 and $\pi$; it comprises the measurements. As there is a large number of views for the reconstruction, we minimize the least-squares error (30) for the reconstruction. This is done for different discretizations of the forward model using KBWFs with different taper parameters. Therefore, the quality of the reconstructed image depends on how well the discretization scheme represents the imaging operator, as shown in Fig. 5(a). We also compute the SNR in the transform domain (Fig. 5(b)). The results validate the importance of using KBWFs with optimized parameters in order to improve the reconstruction performance. We also repeated those experiments with measurements corrupted by additive Gaussian noise with different noise levels $(10 \mathrm{~dB}$, $20 \mathrm{~dB}, 30 \mathrm{~dB})$. The results suggest that using KBWF with the proposed parameters results in better performance. The SNR of the reconstructions was improved by close to $3 \mathrm{~dB}$ with respect to $\alpha=5$.

For the reconstruction of X-ray differential phase-contrast tomograms, it was shown in [7] that using cubic B-splines results in better performance than using KBWFs, with the parameters chosen as in [19]. Here, we compare the performance of three basis functions for the phantom with size $(2,048 \times 2,048)$ pixels. The projection operator is computed using KBWFs with the parameter proposed in [11] $(\alpha=10.4)$ and with the parameter suggested in Fig. $5(\alpha=7.95)$; furthermore, we also perform the comparison with cubic B-splines. The computed SNR shown in Table I suggests that the proposed parameter provides a significantly better performance in computing the projection operator in comparison with the others.

We conclude that a KBWF with the proposed parameters improves the performance of the discretization scheme in comparison with [11], [19]. In addition, its performance is as good as that of cubic B-splines in terms of quality, while its isotropy allows for a drastic reduction in its computational costs.

\section{CONCLUSION}

The projections of isotropic functions are independent of the viewing angle. Therefore, they are attractive candidates as generating functions of principal shift-invariant spaces for discretizing the imaging operators. The generalized Kaiser-Bessel window function is a member of this family that is widely used. In this paper, we proposed a measure to determine the performance of a basis function for the discretization scheme. Furthermore, we suggested a method to optimize the parameters of the KBWF based on this measure. By numerical experiments, we confirmed that using the proposed method improves the performance of the discretization scheme.

\section{APPENDIX}

\section{PROOF OF THEOREM 2}

For the proof, we recall the following theorem.

Theorem 5 [26] (J.L. Griffith): Let $v>-1 / 2$ and $1 / p+$ $1 / q=1$. Let $f$ be an even entire function of exponential type 1. If $1<p \leq 2$ and $t^{\nu+1 / 2} f(t) \in L^{p}(0, \infty)$, then $f$ can be represented by

$$
f(z)=\int_{0}^{1}(x z)^{-v} J_{v}(x z) \phi(x) d x(z \in \mathbb{C}),
$$

with $x^{-v-1 / 2} \phi(x) \in L^{q}(0,1)$. Conversely, if $f$ has this representation and $x^{-v-1 / 2} \phi(x) \in L^{p}(0,1), 1<p \leq 2$, then $f$ is an even entire function of exponential type 1 such that $t^{\nu+1 / 2} f(t) \in L^{q}(0, \infty)$.

We prove Theorem 2 using a proof by contradiction. We suppose that there is a compactly supported isotropic function $\phi$ that satisfies the partition-of-unity condition. Then, using Jensen's theorem, we obtain a contradiction.

Without loss of generality, let us assume that $\phi(\mathbf{x})=0$, for $\|\mathbf{x}\| \geq 1$. We have the following:

- The function $\phi$ is isotropic, so its Fourier transform is the Hankel transform of the function $\bar{\phi}(x)=\phi(\|\mathbf{x}\|)$ with $x=\|\mathbf{x}\|$. We write

$$
\mathcal{F}\{\phi\}(\omega)=2 \pi \int_{0}^{\infty} x \phi(x) J_{0}(\|\omega\|) \mathrm{d} x .
$$

- We define

$$
f(z)=2 \pi \int_{0}^{\infty} x \phi(x) J_{0}(z) \mathrm{d} x,
$$

so $f(\|\omega\|)=\mathcal{F}\{\phi\}(\omega)$. According to Theorem 5 (with $v=0$ ),

$$
f(z)=\int_{0}^{\infty} \psi(x) J_{0}(z x) \mathrm{d} x,
$$

where $\psi(x)=2 \pi x \bar{\phi}(x)$. Since $x^{-\frac{1}{2}} \psi(x) \in L^{2}(0,1)$, $f$ is an even entire function of exponential type 1 .

- Satisfying the partition of unity is equivalent to having the equality in the Fourier domain

$$
\widehat{\phi}(2 \pi \mathbf{n})=\delta[\mathbf{n}],
$$


where $\mathbf{n} \in \mathbb{Z}^{2}$ and $\delta$ is the 2D Kronecker-delta function. It means that the set of zeros of $f(z)$ is $\left\{z=2 \pi\|\mathbf{n}\|, \forall \mathbf{n} \in \mathbb{Z}^{2} \backslash\{\mathbf{0}\}\right\}$. Therefore,

$$
n(R) \geq c R^{2},
$$

where $n(R)$ is the number of zeros in the circle with radius $R$ and $c$ is a positive constant.

- Jensen's theorem implies the inequality

$$
\int_{0}^{R} \frac{n(t)}{t} \mathrm{~d} t \leq \max _{|z|=R} \log |f(z)| .
$$

This inequality restricts the number of zeros inside the disc. We have that

$$
\begin{aligned}
n(R / 2) \log 2 & =\int_{R / 2}^{R} \frac{n(R / 2)}{t} \mathrm{~d} t \\
& \leq \int_{R / 2}^{R} \frac{n(t)}{t} \mathrm{~d} t \\
& \leq \max _{|z|=R} \log |f(z)| .
\end{aligned}
$$

- Since $f$ is of exponential type 1 , it implies that $|f(z)| \leq A e^{|z|}$. Therefore,

$$
\max _{|z|=R} \log |f(z)| \leq C R,
$$

where $C$ is a positive constant.

- Equations (36), (38), and (39) imply that

$$
\begin{aligned}
c(R / 2)^{2} \log 2 & \leq n(R / 2) \log 2 \\
& \leq \max _{|z|=R} \log |f(z)| \\
& \leq C R .
\end{aligned}
$$

Taking $R$ sufficiently large, we reach a contradiction.

\section{REFERENCES}

[1] J. P. Oliveira, J. M. Bioucas-Dias, and M. A. T. Figueiredo, "Adaptive total variation image deblurring: A majorization-minimization approach," Signal Process., vol. 89, no. 9, pp. 1683-1693, 2009.

[2] M. V. Afonso, J. M. Bioucas-Dias, and M. A. T. Figueiredo, "Fast image recovery using variable splitting and constrained optimization," IEEE Trans. Image Process., vol. 19, no. 9, pp. 2345-2356, Sep. 2010.

[3] M. V. Afonso, J. M. Bioucas-Dias, and M. A. T. Figueiredo, "An augmented Lagrangian approach to the constrained optimization formulation of imaging inverse problems," IEEE Trans. Image Process., vol. 20, no. 3, pp. 681-695, Mar. 2011.

[4] S. Ramani and J. A. Fessler, "Parallel MR image reconstruction using augmented Lagrangian methods," IEEE Trans. Med. Imag., vol. 30, no. 3, pp. 694-706, Mar. 2011.

[5] S. Ramani and J. A. Fessler, "A splitting-based iterative algorithm for accelerated statistical X-ray CT reconstruction," IEEE Trans. Med. Imag., vol. 31, no. 3, pp. 677-688, Mar. 2012.

[6] J. M. Fadili and G. Peyré, "Total variation projection with first order schemes," IEEE Trans. Image Process., vol. 20, no. 3, pp. 657-669, Mar. 2011.

[7] M. Nilchian, C. Vonesch, P. Modregger, M. Stampanoni, and M. Unser, "Fast iterative reconstruction of differential phase contrast X-ray tomograms," Opt. Exp., vol. 21, no. 5, pp. 5511-5528, 2013.

[8] Y. Wang, J. Yang, W. Yin, and Y. Zhang, "A new alternating minimization algorithm for total variation image reconstruction," SIAM J. Imag. Sci., vol. 1, no. 3, pp. 248-272, 2008.

[9] E. J. Candès and F. Guo, "New multiscale transforms, minimum total variation synthesis: Applications to edge-preserving image reconstruction," Signal Process., vol. 82, no. 11, pp. 1519-1543, 2002.

[10] L. Ritschl, F. Bergner, C. Fleischmann, and M. Kachelriess, "Improved total variation-based CT image reconstruction applied to clinical data," Phys. Med. Biol., vol. 56, no. 6, pp. 1545-1561, 2011.
[11] R. M. Lewitt, "Multidimensional digital image representations using generalized Kaiser-Bessel window functions," J. Opt. Soc. Amer. A, vol. 7, no. 10, pp. 1834-1846, 1990.

[12] R. Marabini, E. Rietzel, R. Schroeder, G. T. Herman, and J. M. Carazo, "Three-dimensional reconstruction from reduced sets of very noisy images acquired following a single-axis tilt schema: Application of a new three-dimensional reconstruction algorithm and objective comparison with weighted backprojection," J. Struct. Biol., vol. 120, no. 3, pp. 363-371, 1997.

[13] A. P. Leis et al., "Cryo-electron tomography of biological specimens," IEEE Signal Process. Mag., vol. 23, no. 3, pp. 95-103, May 2006.

[14] J.-J. Fernández, D. Gordon, and R. Gordon, "Efficient parallel implementation of iterative reconstruction algorithms for electron tomography," J. Parallel Distrib. Comput., vol. 68, no. 5, pp. 626-640, 2008.

[15] A. Ziegler, T. Köhler, T. Nielsen, and R. Proksa, "Efficient projection and backprojection scheme for spherically symmetric basis functions in divergent beam geometry," Med. Phys., vol. 33, no. 12, pp. 4653-4663, 2006.

[16] A. Ziegler, T. Nielsen, and M. Grass, "Iterative reconstruction of a region of interest for transmission tomography," Med. Phys., vol. 35, no. 4, pp. 1317-1327, 2008.

[17] T. Köhler, B. Brendel, and E. Roessl, "Iterative reconstruction for differential phase contrast imaging using spherically symmetric basis functions," Med. Phys., vol. 38, no. 8, pp. 4542-4545, 2011.

[18] Q. Xu, E. Y. Sidky, X. Pan, M. Stampanoni, P. Modregger, and M. A. Anastasio, "Investigation of discrete imaging models and iterative image reconstruction in differential X-ray phase-contrast tomography," Opt. Exp., vol. 20, no. 10, pp. 10724-10749, 2012.

[19] S. Matej and R. M. Lewitt, "Practical considerations for 3D image reconstruction using spherically symmetric volume elements," IEEE Trans. Med. Imag., vol. 15, no. 1, pp. 68-78, Feb. 1996.

[20] A. Yendiki and J. A. Fessler, "A comparison of rotation- and blob-based system models for 3D SPECT with depth-dependent detector response," Phys. Med. Biol., vol. 49, no. 11, pp. 2157-2168, 2004.

[21] M. Unser, "Sampling-50 years after Shannon," Proc. IEEE, vol. 88, no. 4, pp. 569-587, Apr. 2000.

[22] A. Entezari, D. Van De Ville, and T. Moller, "Practical box splines for reconstruction on the body centered cubic lattice," IEEE Trans. Vis. Comput. Graphics, vol. 14, no. 2, pp. 313-328, Mar./Apr. 2008.

[23] T. Blu and M. Unser, "Approximation error for quasi-interpolators and (multi-)wavelet expansions," Appl. Comput. Harmon. Anal., vol. 6, no. 2, pp. 219-251, 1999.

[24] C. DeBoor, R. A. DeVore, and A. Ron, "Approximation from shiftinvariant subspaces of $L_{2}\left(\mathbb{R}^{d}\right)$," Trans. Amer. Math. Soc., vol. 341, no. 2, pp. 787-806, 1994.

[25] F. Natterer, The Mathematics of Computerized Tomography. New York, NY, USA: Wiley, 1986.

[26] N. B. Andersen, "On Fourier and Hankel sampling," Austral. Math. Soc. Gazette, vol. 33, no. 1, pp. 44-46, 2006

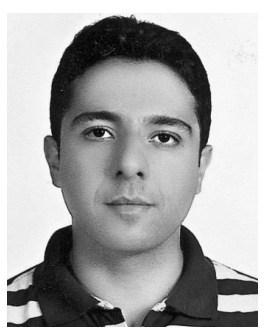

Masih Nilchian received the M.S. degree in electrical engineering with a minor in communication system from the Sharif University of Technology, Tehran, Iran, in 2008, and the Ph.D. degree from the Biomedical Imaging Group, Swiss Federal Institute of Technology, Lausanne, Switzerland, under the direction of Prof. M. Unser, in 2015. He was involved in the applications of information and coding theory in biology and bioinformatics project with the Information System and Security Laboratory, Sharif University. From 2008 to 2010, he was a Research Officer with the Malaysian institute of microelectronic systems (MIMOS) Laboratory, University Technology Malaysia. He is currently a Post-Doctoral Researcher with the Biomedical Imaging Group. His main research area is in phase retrieving in differential phase contrast and phase contrast X-ray computed tomography. He has interest in the use of spline for image processing, information theory, coding theory, wavelet and multiresolution algorithms, and iterative methods for reconstruction. 


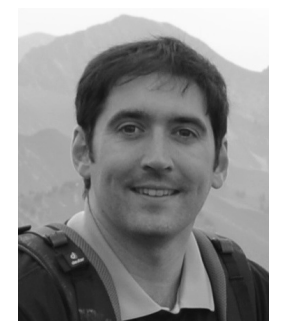

John Paul Ward received the B.S. degree in mathematics from the University of Georgia, Athens, in 2005, and the Ph.D. degree in mathematics from Texas A\&M University, College Station, in 2010 He is currently a Post-Doctoral Researcher with the Biomedical Imaging Group, Swiss Federal Institute of Technology, Lausanne, Switzerland.

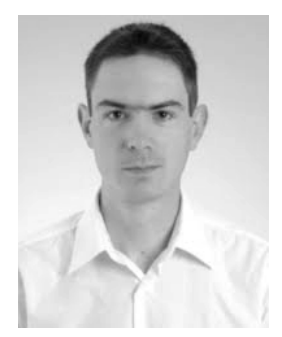

Cédric Vonesch was born in Obernai, France, in 1981. He received the M.S. degree from the Swiss Federal Institute of Technology in Lausanne (EPFL), Switzerland, in 2004, and the Ph.D. degree from the Biomedical Imaging Group, EPFL, under the direction of Prof. M. Unser, in 2009. He then joined the Program in Applied and Computational Mathematics with Princeton University for a post-doctoral position. Since 2012, he has been with Biomedical Imaging Group, EPFL. His primary research interests are inverse problems in the imaging sciences and multiresolution (wavelet-based) methods. He has worked on applications to fluorescence and electron microscopy, and seismic imaging.

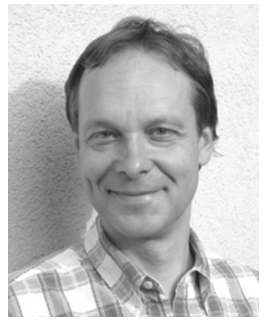

Michael Unser (M'89-SM'94-F'99) received the M.S. (summa cum laude) and Ph.D. degrees in electrical engineering from the École Polytechnique Fédérale de Lausanne (EPFL), Switzerland, in 1981 and 1984, respectively. From 1985 to 1997, he was a Scientist with the National Institutes of Health, Bethesda, USA. He is currently a Full Professor and the Director of the Biomedical Imaging Group at EPFL. His main research area is biomedical image processing. He has a strong interest in sampling theories, multiresolution algorithms, wavelets, and the use of splines for image processing. He has authored 200 journal papers on those topics, and is one of the institute for scientific information (ISI's) Highly Cited authors in Engineering. He is currently a member of the Editorial Boards of Foundations and Trends in Signal Processing, and Sampling Theory in Signal and Image Processing. He is a fellow of the European association for signal processing (EURASIP) and a member of the Swiss Academy of Engineering Sciences. He co-organized the first IEEE International Symposium on Biomedical Imaging and was the Founding Chair of the Technical Committee of the IEEE-SP Society on Bio Imaging and Signal Processing. He received the 1995 and 2003 Best Paper Awards, the 2000 Magazine Award, and the two IEEE Technical Achievement Awards (2008 SPS and 2010 EMBS). He has held the position of Associate Editor-inChief of the IEEE TRANS ACTIONS ON MEDICAL IMAGING (2003-2005), and has served as Associate Editor for the IEEE TRANSACTIONS ON MEDICAL IMAGING (1999-2002; 2006-2007), the IEEE TRANSACTIONS ON IMAGE Processing (1992-1995), and the IEEE Signal Processing LetTers (1994-1998). 